
(NSN)

\title{
Ascorbic Acid Supplementation Attenuates Hippocampal Neuronal Damage in Orchiectomized Rats
}

\author{
Taiwo A. Abayomi ${ }^{1}$, Olorunfemi S. Tokunbo ${ }^{1}$, Mercy Oyetunti ${ }^{1}$, \\ Emmanuel O. Yawson ${ }^{2}$, Olawale A. Abayomi ${ }^{3}$, Oluwayemisi D. Ogunrinde ${ }^{1}$ \\ ${ }^{1}$ Department of Anatomy, Faculty of Basic Medical Sciences, Osun State University, Osogbo, Nigeria \\ ${ }^{2}$ Department of Anatomy, Faculty of Basic Medical Sciences, Redeemers University, Ede, Nigeria \\ ${ }^{3}$ Department of Radiology, Faculty of Clinical Sciences, Ekiti State University, Ado Ekiti, Nigeria
}

Received:

April 2021

Accepted: June 2021

\begin{abstract}
Understanding the bidirectional relationship in the cellular and molecular mechanisms associated with testosterone deprivation and cognitive activities has become a high-priority goal. Testosterone has been shown to have effects in the nervous system, ranging from targeting gene expression to modulating neurotransmission. This study therefore evaluated the modulatory role of ascorbic acid in the hippocampus of orchiectomized rats. Twenty-one adult male Wistar rats with an average weight of $170 \mathrm{~g} \pm 10 \mathrm{~g}$ were randomly assigned into three groups of seven rats each; the control, orchiectomized (orchiectomy+flutamide, $11 \mathrm{mg} / \mathrm{kg}$ body weight, bw), and ascorbic acid (orchiectomy+flutamide, 11 $\mathrm{mg} / \mathrm{kg}$ bw + ascorbic acid, $100 \mathrm{mg} / \mathrm{kg}$ bw). Treatment was by oral gavage and lasted for 30 days. Nitrosative stress and neuroinflammatory analysis, hormonal, histological and immunohistochemical expression of astrocytes in the hippocampus were examined. Results showed significantly increased expression of acetylcholinesterase, inducible nitric oxide synthase, and tumour necrotic factor-alpha in the hippocampus of orchiectomized animals. There was altered cytoarchitectural morphology evidenced by reduced Nissl profiles in neuronal axons and dendrites, which corresponded to apoptotic changes, and increased expression of reactive astrocytes suggesting neuronal damage. Nitrosative stress and inflammatory perturbations were well modulated in animals treated with ascorbic acid with unaltered hippocampal morphology. The results indicated that decline in brain androgen activities caused inflammatory and oxidative stress-driven alterations in the hippocampus, while ascorbic acid supplementation offered therapeutic value by modulating neurochemicals and scavenging free radicals in the hippocampus.
\end{abstract}

Key words: Anti-inflammation, Ascorbic acid, Hippocampus, Orchiectomy, Testosterone

\section{INTRODUCTION}

Neurodegeneration, and testosterone deficiency syndrome is known to affect millions of people worldwide. There is increasing evidence from clinical and epidemiological research suggesting that testosterone deficiency and Alzheimer's disease; associated with dementia are linked (Okun et al. 2004). Testosterone has been reported to play important roles in neuronal and astrocyte health, formation of synaptic plasticity and cognitive function
(Vicencio et al. 2011; Jacome et al. 2016; Akinola and Gabriel 2018).

Androgen receptors are expressed across brain regions, some of which includes the corticohippocampal areas and basal ganglia, these receptors have been associated with learning and

Correspondence: Taiwo A. Abayomi, PhD, Department of Anatomy, Faculty of Basic Medical Sciences, Osun State University, PMB 4494, Osogbo, Osun State, Nigeria.. taiwo.abayomi@uniosun.edu.ng, +2349130913964 
cognition. Hence, testosterone deficiency affect cognition and elicit Alzheimer-like pathology (Akinola and Gabriel 2018).

Orchiectomy also named orchidectomy, and sometimes shortened as orchi, involves the surgical removal of one or both testicles. The removal of both testicles (bilateral orchiectomy) is commonly performed as part of sex reassignment surgery for transgender women, or as a means for managing advanced cases of prostate cancer (testosterone ablation therapy), as well as testicular cancer (Gooren and Asscheman 2014).

Testosterone plays a major role in sexual desire, erection and secondary sexual characteristics. It facilitates various metabolic processes occurring in the body. Therefore, deficiency can be associated with metabolic dysfunctions (Bagatell and Bremner 1996). About $80 \%$ of the total testosterone in male individuals binds to sex hormone binding globulin and acts as a reserve source. Free testosterone and albumin bound testosterone are biologically active representing $20 \%$ of total testosterone (Morris and Channer 2012). Andropause, the gradual decline of testosterone levels associated with aging may be due to reduction of Leydig cell mass, testicular circulation, hypothalamic gonadotropic releasing hormone $(\mathrm{GnRH})$ and pituitary gonadotropins, and is a prevalent and serious aging related problem (Veldhuis et al. 2009). Decreased androgen levels associated with aging is referred as late-onset hypogonadism. It manifests by (i) somato-vegetative conditions, including fatigue, insomnia, osteopenia, sarcopenia and visceral obesity, (ii) psychological conditions, including disturbed sense of well-being, mood disorders and anxiety and (iii) sexual conditions, including erectile dysfunction and decreased libido (Namiki et al. 2008; Bhasin et al. 2010) .

Bilateral orchiectomy in males reduce the level of circulating testosterone which leads to alterations in sexual, physical, social and cognitive functions (Kluger et al. 2020). Moreover, studies have shown that blood testosterone levels in healthy males with intact gonads drop gradually as they advance in age (Chen et al. 2001; Wiltink et al. 2009; Beattie et al. 2015; Kluger et al. 2020). In addition, male circulating oestradiol level is from the aromatization of gonadal testosterone, therefore, depleted free testosterone may consequently result in depleted free oestradiol level (Ferrini and Barrett-Connor 1998). Furthermore, aside bilateral orchiectomy, male population with prostatic condition choose androgen deprivation therapy to bring about andropause. This is a treatment that suppresses plasma androgens with the use of antiandrogens in addition to long-acting GnRH agonists (Ayub and Levell 1990). Despite achievements from this method, one of its reported concerns is in relation to the effects of such artificial manipulation on the endocrine functions and the resultant effects on cortico-hippocampal (learning and cognitive) functions in the affected male population (Akinola and Gabriel 2018).

Vitamins are viewed as natural sources for treating neurological conditions, given that their deficiencies are known to initiate neurodegenerative conditions. Ascorbic acid (vitamin C) is a water-soluble micronutrient required for multiple biological functions. Ascorbic acid is a co-factor for several enzymes participating in the post-translational hydroxylation of collagen, biosynthesis of carnitine, conversion of the neurotransmitter dopamine to norepinephrine, peptide amidation and in tyrosine metabolism. In addition, vitamin $\mathrm{C}$ is an important regulator of iron uptake; it reduces ferric $\mathrm{Fe}^{3+}$ to ferrous $\mathrm{Fe}^{2+}$ ions, thus promoting dietary non-haem iron absorption from the gastrointestinal tract, and stabilizes iron-binding proteins. Most animals are able to synthesize vitamin $\mathrm{C}$ from glucose, but humans, other primates, guinea pigs and fruit bats lack the last enzyme, gulonolactone oxidase involved in the synthesis of vitamin $\mathrm{C}$ and so require supplementation or the presence of the vitamin in their diet.

This study was to investigate the putative effect of ascorbic acid on cellular and molecular brain changes that underlie some behavioural and cognitive perturbations in the hippocampus of orchiectomized rats.

\section{MATERIALS AND METHODS}

\section{Animal Care and Ethical Approval}

Twenty-one adult male Wistar rats with an average weight of $170 \pm 10 \mathrm{~g}$ were used for this study. The rats were housed in the animal holding of the College of Health Sciences, Osun State University, and had access to rat chow and water ad libitum. All protocols on animal handling strictly followed the guidelines of Institutional Animal Care and Use Committee (IACUC) as approved by the College of Health Sciences Ethics Review Committee, Osun State University, Nigeria (UNIOSUNHREC 2021/020)

\section{Procurement and Reconstitution}

Flutamide (Cas. NO: 13311-84-7) was obtained from Akol Pharmaceutical store, Osogbo, while ascorbic acid (Cas. NO: 50-81-7) was purchased from Sigma (Aurich, Germany); they were dissolved in distilled water. These solutions were freshly prepared on the first day of administration and kept at $4{ }^{\circ} \mathrm{C}$ until use.

\section{Animal Grouping and Treatment}

The animals were randomly divided into three groups comprising seven rats: Control (normal saline); orchiectomized group (orchi+flutamide, $11 \mathrm{mg} / \mathrm{kg}$ body weight, bw); ascorbic acid group (orchi+flutamide, $11 \mathrm{mg} / \mathrm{kg}$ bw + ascorbic acid, 100 $\mathrm{mg} / \mathrm{kg} \mathrm{bw}$ ). Flutamide and ascorbic acid were 
administered by oral gavages 24 hours after orchiectomy was performed, and subsequently, daily at 9:00 $\mathrm{h}$ for a total of 30 days (Yawson and Akinola 2021).

\section{Operative Technique for Orchiectomy}

Orchiectomy was performed by using the method described by Idris (2012) in a sterile environment. Summarily, anaesthesia was induced by intraperitoneal injection of ketamine hydrochloride (50 $\mathrm{mg} / \mathrm{kg}$ ). After the onset of anaesthesia, the animals were placed under infrared lamp to prevent heat loss. An electric clipper was then used to shave the fur bilaterally over the ventral side of the scrotum to expose the skin. The exposed skin was swabbed with $70 \%$ ethanol followed by sterile phosphate buffered saline (PBS). A single incision that penetrated the skin and cremaster muscle was made on the ventral side of the scrotum $(1.6 \mathrm{~cm})$ using sterile scalpel. The testicular fat pad was gently pulled through the incision using sterile blunt forceps. Testicular content were carefully exposed and a single ligature was made around the blood vessels to prevent bleeding. The caudal epididymis and caput epididymis were severed from the testis after which the testis was removed gently by severing blood vessels with a small scissors. The remaining content of the testicular sac were carefully replaced and the skin was closed using metal clips. Anaesthesia was then reversed by intraperitoneal injection of atipamezole hydrochloride (1 $\mathrm{mg} / \mathrm{kg})$. Administration of experimental treatment was delayed for 24 hours after surgery to allow full recovery from anaesthesia.

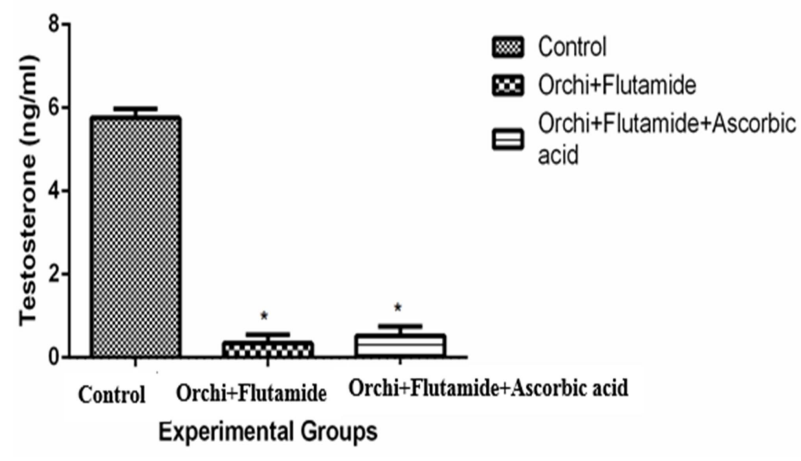

Fig. 1: Concentration of serum testosterone level across the experimental animals. The values are expressed as Mean \pm SEM. * significant in comparison to the control group

\section{Animal Sacrifice}

After completion of treatments, the animals were anaesthetized with $50 \mathrm{mg} / \mathrm{kg}$ of ketamine hydrochloride. Venous blood was taken from a large vein in the hind limb of the rats for hormonal assay after which the brain was quickly dissected out, placed on a pre-chilled metal plate resting on crushed ice for the separation of the hippocampus. The right hemisphere of the hippocampus was excised and fixed in $10 \%$ buffered neutral formalin for 2 days for histological and immunohistochemical evaluation, while the left hemisphere was preserved in $0.1 \mathrm{M}$ of PBS for biochemical analysis.

\section{Histological Tissue Processing and Staining}

Neutral buffered formalin-fixed hippocampal tissues were embedded in paraffin, and coronal sections (5 $\mu \mathrm{m})$ were taken serially. The sections were then processed for general histology (haematoxylin and eosin) using the method described by Spencer et al. (2012), and cresyl violet stain to reveal Nissl substances in by the method of Pilati et al. (2008).

\section{Hormone Measuring Assay}

Blood was collected into a heparinized bottle and serum was immediately collected by centrifugation (4000 rpm at $4^{\circ} \mathrm{C}$ ) and stored at $-20^{\circ} \mathrm{C}$. Serum level of testosterone was determined using ELISA kit according to the manufacturer's instructions, and all samples were tested in triplicate. ELISA Kit was obtained from Monobind Inc. Lake forest, CA, USA (Testosterone Cat \#: 3633-300).

\section{Biochemical Assays}

Determination of acetylcholinesterase (AChE), inducible nitric oxide synthase (iNOS), nitric oxide and tumour necrosis factor (TNF- $\alpha$ ) activities were evaluated on the homogenized hippocampal tissues using the spectrophotometric technique. AChE (ab138871), iNOS (ab253219), nitric oxide (ab65328) assay kits were procured from Abcam, USA; and TNF-a assay kit (RAB0477-1KT) was procured from MilliporeSigma, USA. Hippocampal section from rats across groups were weighed and pulverized in 0.25 $M$ sucrose (Sigma) with the aid of an automated homogenizer at $4^{\circ} \mathrm{C}$. The supernatants were aspirated into plain labelled glass cuvette placed in ice. The different biochemical markers were assayed according to the manufacturer's instruction in the assay kit packs.

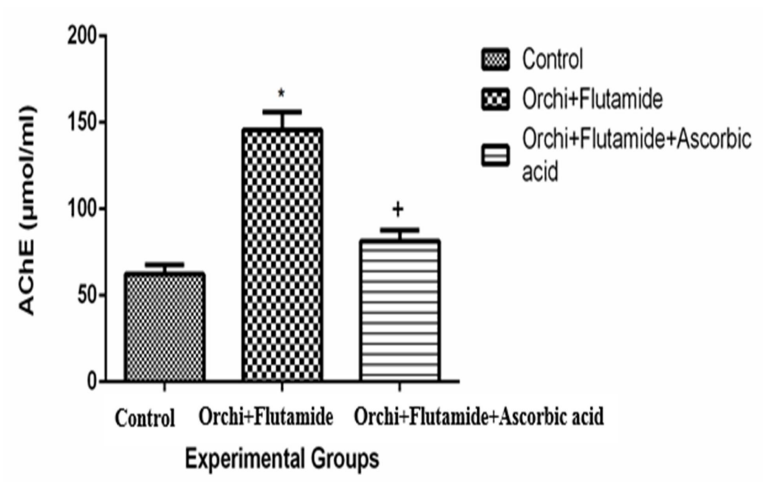

Fig. 2: Activity of Acetylcholinesterase (ACHE) in the hippocampus across the experimental animals. The values are expressed as Mean \pm SEM. * significant in comparison to the control group; + significant in comparison with orchi+flutamide group. 


\section{Immunohistochemical Study}

Formalin-fixed tissue sections were deparaffinized in xylene, rehydrated with descending grades of alcohol and heated in microwave for $10 \mathrm{~min}$ to retrieve antigen. Sections were incubated in $0.3 \%$ hydrogen peroxide for $10 \mathrm{~min}$ to abolish endogenous peroxidase activity, before blocking with $5 \%$ bovine serum albumin for $30 \mathrm{~min}$. Slides were incubated with primary antibody (1:500 monoclonal mouse antiGFAP) for $30 \mathrm{~min}$, washed twice in Tris buffered saline (TBS) for 3 min and incubated with avidin biotinylated conjugated antibodies (ABC kit, 1:200) for $30 \mathrm{~min}$. Finally, sections were developed with diaminobenzidine. Slides were counterstained with Mayer's haematoxylin, after which dehydration, clearing and mounting were done.

\section{Statistical Analysis}

GraphPad Prism version 7.0 was used for all statistical analyses. All data were expressed as Mean \pm Standard Error of Mean (SEM) and differences among the groups were analysed by one-way analysis of variance. Turkey's correction was used to adjust for multiple comparisons while $P$ value at $<0.05$ was considered to be statistically significant.

\section{Photomicrography}

Photomicrographs were obtained using Olympus binocular research microscope (Olympus, New Jersey, USA) and a 5.0MP Amscope Camera (Amscope inc, USA). Image $J$ was used to quantify the expression of glial fibrillary acidic protein (GFAP).

\section{RESULTS}

\section{Quantitative Analysis}

Orchiectomized rats (orchi+flutamide) showed reduced serum testosterone level $(0.344 \pm 0.2002)$ which was significant $(p<0.05)$ when compared to the control group. Rats treated with ascorbic acid after orchiectomy and flutamide administration (orchi+flutamide+ascorbic acid) also had significantly reduced serum testosterone level $(0.4220 \pm 0.3112)$ when compared with the control group (Fig. 1)

The orchiectomized rats had significant increased activities of AChE (145.8 \pm 10.30$)$ when compared to the control group (62.20 \pm 4.27$)$. Ascorbic acid group expressed reduced levels of AChE $(81.40 \pm 6.32)$ which was significant when compared to orchiectomized rats $(p<0.05)$ (Fig. 2).

Inducible nitric oxide synthase activity increased significantly in orchiectomized animals (24.68 \pm 0.9800 ) when compared with the control group (11.48 \pm 1.332 ) and animals treated with ascorbic acid $(13.20 \pm 1.934)$ (Fig. 3a). The level of nitric oxide increased significantly $(p<0.05)$ in the orchiectomized rats $(311.4 \pm 17.49)$ when compared to the control group (126.2 \pm 12.67) and animals treated with ascorbic acid (139.6 \pm 11.41$)$ (Fig. 3b).

Increased expression of TNF- $\alpha$ was observed in the orchiectomized animals $(177.6 \pm 6.735)$ which was significant $(p<0.05)$ when compared to the control group (83.20 \pm 3.382$)$ and ascorbic acid group (92.80 \pm 4.620 ) (Fig. 4).

\section{Qualitative Analysis}

Haematoxylin and eosin staining showed the general cytoarchitecture of the hippocampus of experimental rats (Fig. 5). The control group and the ascorbic acid group had normal morphological presentation of the hippocampal layers. In these groups, the fine array of cells within the hippocampus could be seen distinctly from the cornu ammonis (CA) to the dentate gyrus. The orchiectomized group showed induced degenerative changes in the hippocampus, characterised by fragmented pyramidal and granule cell layers.

Nissl profile demonstration by cresyl violet stain (Fig. 6) showed the hippocampus of animals treated with ascorbic acid was characterized with normal and densely populated Nissl, well stained and outlined neurons. The orchiectomized group was characterized by chromatolytic changes in the different cell layers with an associated reduction in the cytoplasmic Nissl proteins.

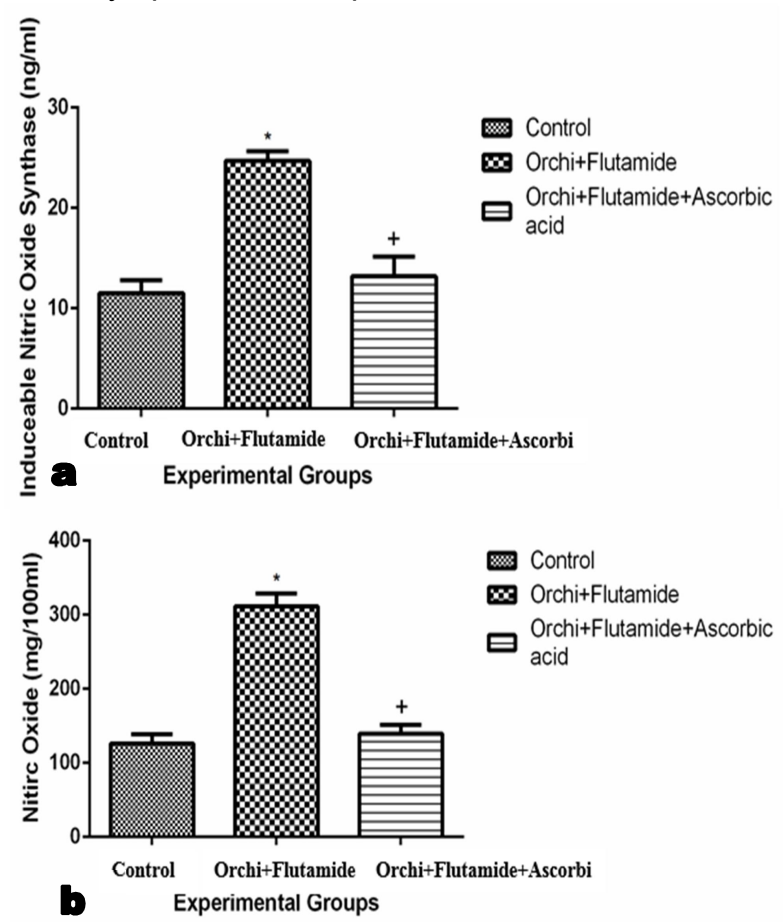

Fig. 3: level of inducible nitric oxide synthase (a) and nitric oxide (b) in the hippocampus across the in the hippocampus across the experimental animals. The values are expressed as Mean \pm SEM. * significant in comparison to the control group; + significant difference in comparison with orchi+flutamide group. 
Astrocyte activity in the hippocampus showed dense staining intensity and an increased number of reactive astrocytes in the orchiectomized group. The staining intensity and astrocyte immunoreaction in the control group and ascorbic acid treated animals were largely similar (Fig. 7).

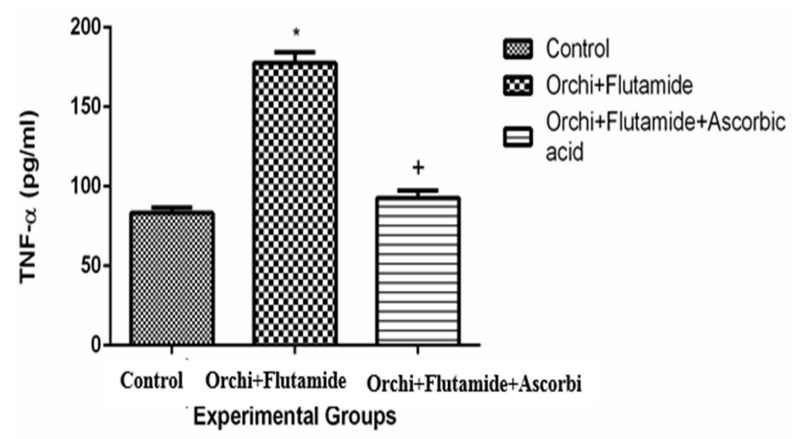

Fig. 4: Expression of tumor necrotic factor- $\alpha$ in the hippocampus across the experimental animals. The values are expressed as Mean \pm SEM. * significant in comparison to the control group; + is the significant in comparison with orchi+flutamide group.

\section{DISCUSSION}

The present study showed that ascorbic acid may change the course of neurological diseases arising from androgen depletion due to its antioxidative and anti-inflammatory effects. In establishing the relationship between circulating levels of testosterone and hippocampal function, the level of serum testosterone in the experimental animals was evaluated. Treatment with flutamide, an androgen receptor antagonist and orchiectomy critically reduced serum testosterone levels. Reduced serum testosterone levels following orchiectomy and flutamide treatment reported in this study lend credence to the fact that treating conditions associated with hormonal pertubations such as prostate cancer, may result in undesirable psychological demerits, indicating that an optimal level of sex steroids is required for brain health. The report of Mellon and Deschepper (1993) supports the current findings: They reported that testosterone loosely bound or unbound to plasma proteins plays vital a role in cognitive performance. This free testosterone which is about $2 \%$ of the total testosterone in serum or plasma enters the brain and augments the activities of endogenously synthesized steroid hormones. Several reports correlated higher levels of bioavailable testosterone with better cognitive performance, superior performance on visual and verbal memory, improved performance in working memory tasks (Janowsky et al. 2000) and improved spatial cognition (Janowsky et al. 1994; Cherrier et al. 2001) compared to men with lower plasma testosterone levels (Yaffe et al. 2002).
Furthermore, studies have also linked testosterone depletion with increased depression and anxiety scores (Dewing et al. 2003), increased risk of developing Alzheimer's and Parkinson diseases.

An imbalance in the cholinergic system, such as reductions of acetylcholine (ACh) in brain structures, reduced densities of nicotinic and muscarinic receptors and overexpression of AChE characteristic of some neurodegenerative disorders like Alzheimer's and schizophrenia. AChE is an important enzyme in the cholinergic system since it is responsible for the hydrolysis and inactivation of $\mathrm{ACh}$ to choline and acetate (Soreq and Seidman 2001). In this study, overexpression of hippocampal AChE was observed in animals that underwent orchiectomy and flutamide treatment, while ascorbic acid supplementation modulated AChE activity. One way by which testosterone modulates cognitive function is by influencing the cholinergic system through an increase in $\mathrm{ACh}$ release and by modulating nicotinic receptors (Damaj 2001; Nakamura et al. 2002). Testosterone depletion occasioned by orchiectomy and flutamide treatment in this study, may have altered the balance in the cholinergic system, leading to an increase in ACHE activity at the cholinergic synapse, reduction in acetylcholine levels, and suboptimal cholinergic neurotransmission. There is evidence that ascorbate is a neuromodulator of cholinergic transmission. The present result indicates that treatment with ascorbic acid reduced the activity of AChE, thereby allowing a possible increase in levels of $\mathrm{ACh}$ in the synaptic cleft. This could be due to redox modulation of nicotinic receptors by ascorbate, or to direct scavenging of reactive oxygen species generated by receptor activation (Rosa et al. 2010; Abayomi et al. 2019).

In this study, androgen depletion orchestrated by orchiectomy and flutamide treatment elevated hippocampal levels of nitric oxide and iNOS significantly when compared to the control group and animals treated with ascorbic acid. Nitric oxide is a bioactive lipophilic molecule that plays different roles in the brain. Nitric oxide as a neurotransmitter in synapse allows brain blood flow and also plays important roles in intracellular signalling. Nitric oxide production majorly requires nitric oxide synthase (NOS), which catalyze the oxidation of L-arginine to yield citrulline and nitric oxide (Avontuur et al. 1996; Beck et al. 1998). Inducible nitric oxide synthase (iNOS) is one of the isoforms of NOS, and several inflammatory stimuli can induce the expression of iNOS in various cell types such as microglial, astrocytes, endothelial cells at the blood-brain barrier and neurons in the central nervous system (CNS). Proper neuronal function requires minimal physiological concentrations of nitric oxide in the CNS, with sustained high nitric oxide levels leading to detrimental effects when it reacts with superoxide anion to form peroxynitrite (May 2000). The findings of this present study would be the results of a lack of 


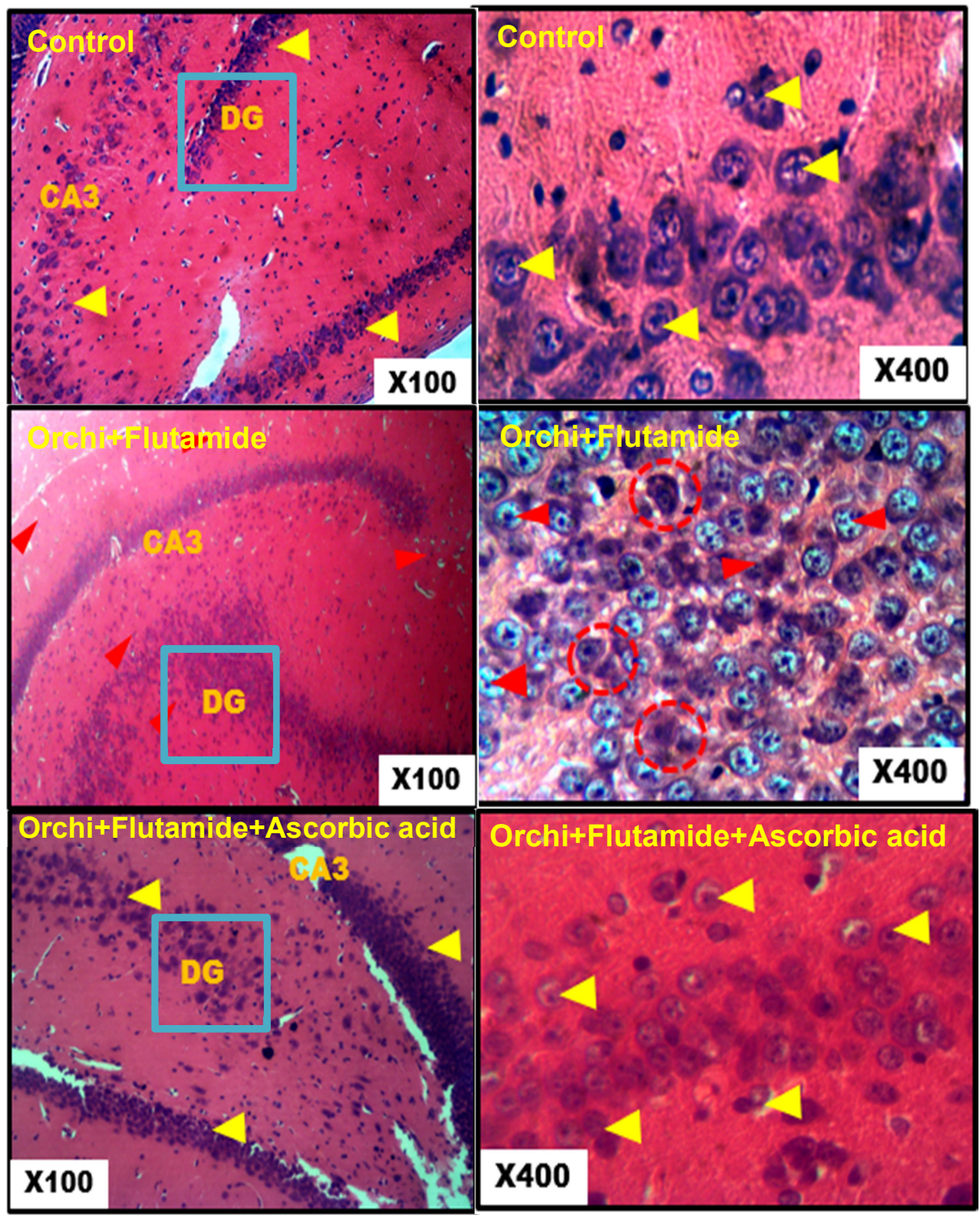

Fig. 5: Photomicrographs of the hippocampus across the various study groups. The dentate gyrus (DG) composed of granule cells, Cornu amonis (CA1-3) containing pyramidal cells can be seen. Haematoxylin and eosin $(\times 100, \times 400)$

control of iNOS expression by neurons and activated astrocytes induced pathologically by an inflammatory response to depleted androgen levels in the hippocampus. High concentrations of nitric oxide, peroxynitrite, and other reactive nitrogen species have been found to correlate with greater severity of neurodegenerative disorders. Moreover, nitrotyrosination has been shown to be a common signature in Alzheimer's disease and the present findings lend credence to evidences suggesting a link between testosterone deficiency and Alzheimer's disease. Furthermore, the hippocampus is very susceptible to nitrative and oxidative damages because of its high oxidative metabolism; this could lead to reversible or irreversible processes. These processes may result in the build-up of modified proteins which may contribute to the onset and progression of neurodegenerative diseases such as multiple sclerosis, Alzheimer's or Parkinson.

The present result also showed that ascorbic acid supplementation inhibited iNOS derived nitric oxide expression in the hippocampus of testosterone deficient rats. This may be as a result of the antiinflammatory and antioxidative properties of ascorbic acid, which may have attenuated the orchiectomy-induced inflammatory reactions from brain immune cells, thereby modulating the expression of neuronal nitric oxide synthase and iNOS. The antiinflammatory activity of ascorbic acid suggests that conditions associated with chronic inflammation can be alleviated.

Neurons when inflamed, produced TNFa, a signalling protein involved in systemic inflammation. Perturbations in TNF production has been implicated in a variety of neurodegenerative diseases. The present result demonstrates that androgen deprivation via orchiectomy acted as inflammatory stimulus that activated the release of TNF. Ascorbic acid treatment attenuated inflammatory responses induced by depleted androgen levels by modulating the expression of TNF- $\alpha$ in the hippocampus.

The inhibition of proinflammatory cytokine expression in the brain, which may be dependent upon activated androgen receptors or the conversion of testosterone into oestradiol by aromatase enzyme, is a key mechanism by which testosterone protects brain cells (Katoh-Semba et al. 1994). Studies have shown that low testosterone is associated with promotion of inflammatory pathways (Aragno et al. 2002) and the 


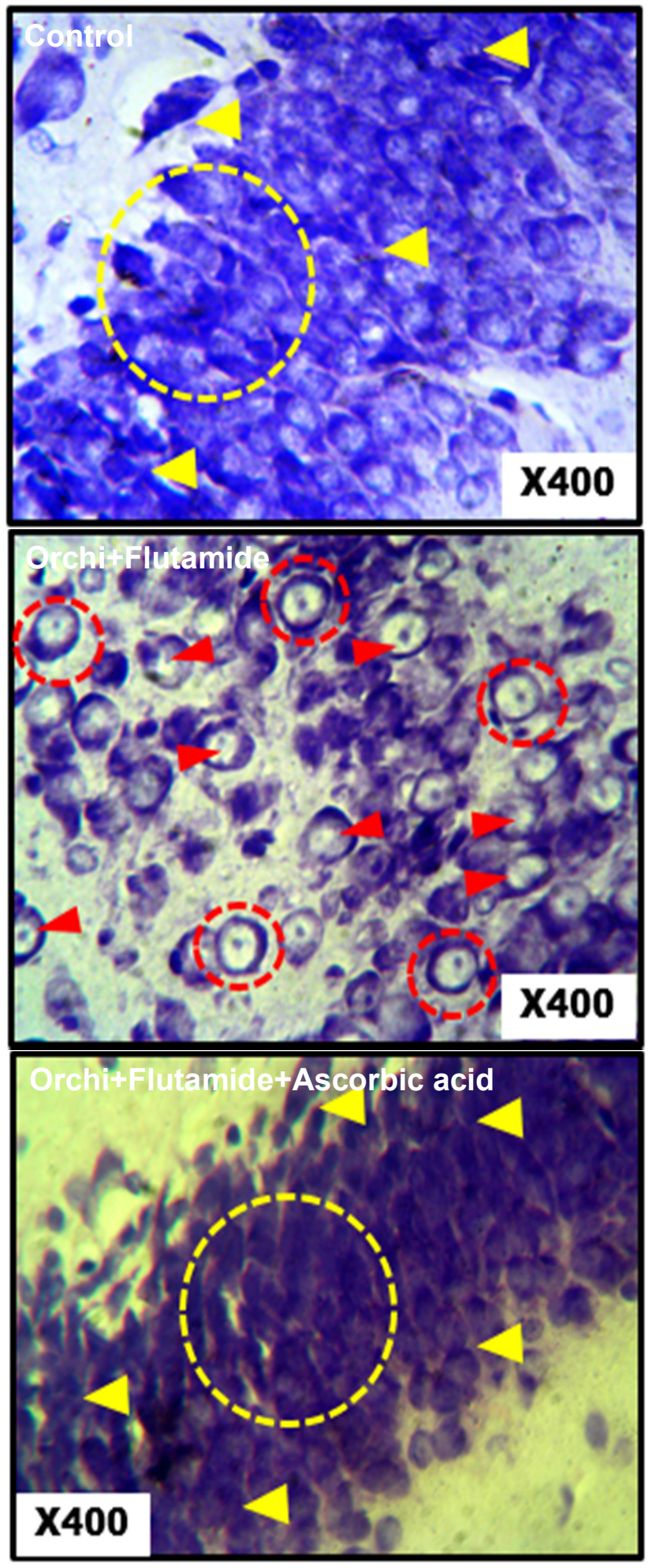

Fig. 6: Photomicrographs of the hippocampus showing the expression of Nissl. Cresyl violet $(\times 400)$

persistence of an inflammatory stimulus has detrimental actions on the nervous system (Benveniste 1998; Streit et al. 2004; Cappellano et al.
2013). Ascorbic acid plays important role in conditions that are linked to inflammatory processes. As an essential micronutrient, it ensures the efficient working of the immune system and provides cellular defence against chronic inflammation.

-Furthermore, the present study reports that orchiectomy elicited the activation of reactive astrocyte. The robust increase in glial fibrillary acidic protein (GFAP) expression in orchiectomized animals suggest that increases in the expression of iNOS, nitric acid and TNFa resulted from classically activated astrocytes, and these findings are consistent with findings from previous studies (Madrigal et al. 2002; Medeiros et al. 2007; Jang et al. 2013). Neural health indices are linked with the activities of astrocytes because they provide structural and functional support for neurons. The function of glia cells, which are major sources of proinflammatory molecules in the CNS are affected by testosterone (Jayaraman et al. 2014) and can display a wide spectrum of immune and inflammatory mediators in the microenvironment that may eventually result in neurotoxic or neuroprotective outcomes (Ridet et al. 1997). It was observed that ascorbic acid supplementation prevented astrocyte activation which is a common signature in brain pathological conditions. Ascorbic acid recycling in the brain which is mediated by interplay of astrocytes and neurons is vital for the maintenance of normal brain function. This interplay involves the uptake of dehydroascorbic acid by astrocytes through glucose transporter, conversion to ascorbic acid, subsequent release from astrocytes, and entry into neurons through the sodium vitamin $\mathrm{C}$ transporters. In pathological conditions where concentrations of NOS and other inflammatory biomarkers are increased, these recycling mechanisms may be compromised, eliciting neuronal toxicity. Hence, ascorbic acid through its antioxidant and anti-inflammatory actions can mediate cellular toxicity resulting from low testosterone levels in the brain.

The morphological demonstration of the hippocampus of orchiectomized animals in this study showed pathological changes characterized by necrotic and chromatolytic neurons. Consistent with findings from this study, decreased Nissl staining intensity is often seen in pathological conditions (Gonzalez-Forero and Moreno-Lopez 2014). Uncontrolled generation of reactive oxygen species is a known cause of necrotic neuronal death which is the hallmark of neurodegenerative diseases (Sunday et al. 2019). Ascorbic acid proved to be protective against low testosterone-induced neuronal damage by maintaining the structural integrity of the hippocampus. 

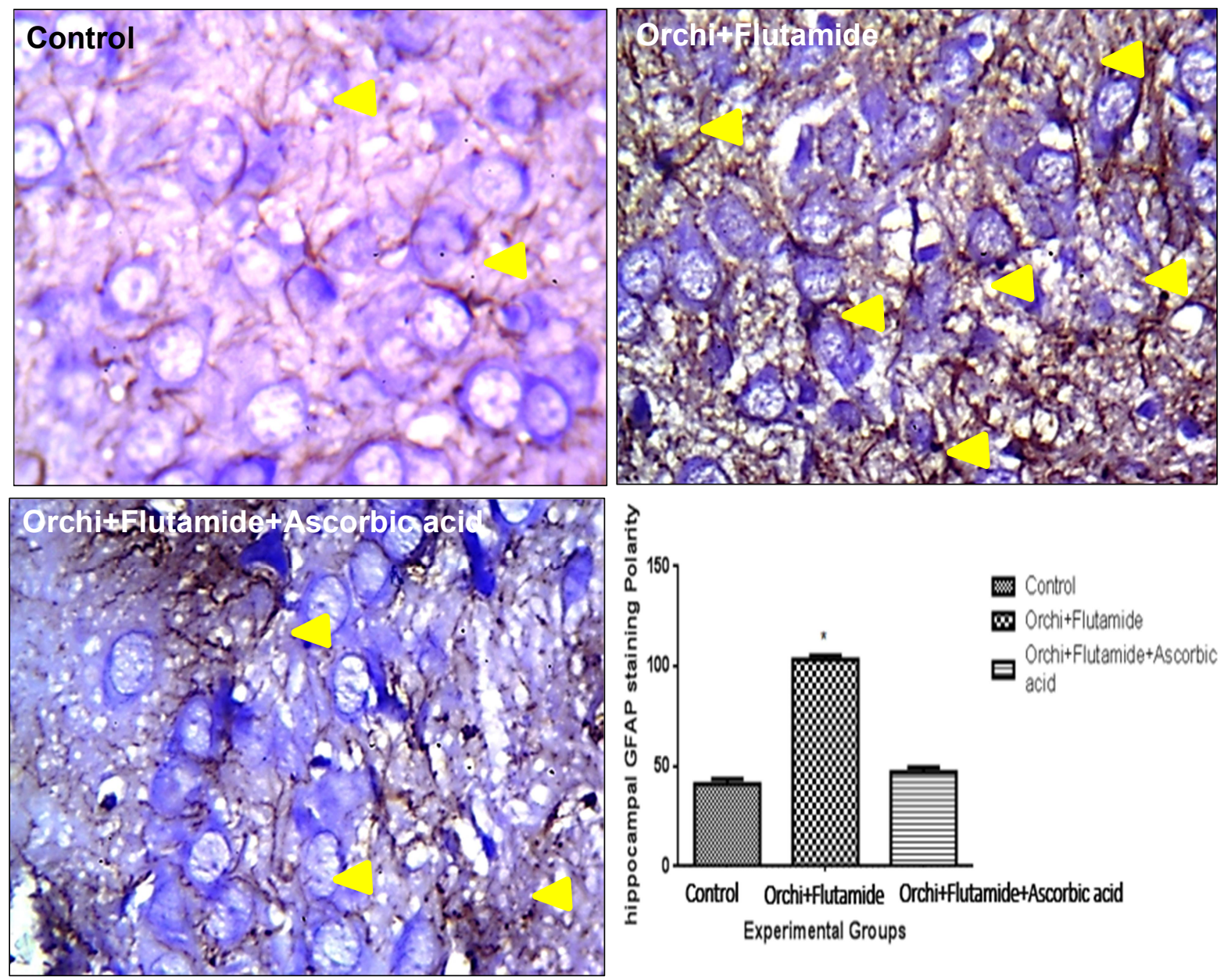

Fig. 7: Photomicrographs of immuno expression of astrocytes in the hippocampus of experimental animals across the study groups. GFAP $(\times 400)$

\section{Conclusion}

Ascorbic acid plays a prominent role in the defense against damages induced by a decline in androgen activities in the brain. Findings from this study shows ascorbic acid as an antioxidant molecule, a neuromodulator of synaptic activity, and as a mediator in inflammatory responses: Thus, this molecule can provide therapeutic benefits in neuropathological conditions associated with testosterone deficiency.

\section{Conflict of Interest}

None declared.

\section{Author Contributions}

TAA: Concept and design of the research, Orchiectomy procedure, and critical revision of the manuscript for intellectual content, Approval of article. OST: Conception and design, Drafting of the manuscript, and performed the orchiectomy procedure.
MO: Acquisition of data, data analysis and interpretation.

EOY: Critical revision of the manuscript for intellectual content,

OAA: Data analysis and interpretation, Approval of final article

ODO: Acquisition of data, Provision of study materials, data analysis and interpretation.

\section{REFERENCES}

Abayomi, T.A., Tokunbo, O.S., Adebisi, B.T., Fadare, M.U., Gbadamosi, I.T., Akinwale, J.O., et al. (2019) Neurobehavioral assessment of the impact of vitamins $C$ and $E$ following acute exposure to sodium azide-induced neurotoxicity. J Environ Toxicol Public Health. 4:15-20.

Akinola, O.B. and Gabriel, M.O. (2018) Neuroanatomical and molecular correlates of cognitive and behavioural outcomes in hypogonadal males. Metab Brain Dis. 33(2):491-505. 
Aragno, M., Mastrocola, R., Brignardello, E., Catalano, M., Robino, G., Manti, R., et al. (2002) Dehydroepiandrosterone modulates nuclear factor-KB activation in hippocampus of diabetic rats. Endocrin. 143(9):3250-3258

Avontuur, J.A., Bruining, H.A. and Ince, C. (1996) Sepsis and nitric oxide. In: Oxygen Transport to Tissue XVII. Springer, Boston, MA. Pp. 551-567.

Ayub, M. and Levell, M.J. (1990) Suppression of plasma androgens by the antiandrogen flutamide in prostatic cancer patients treated with Zoladex, a GnRH analogue. Clin Endocrin. 32(3):329-340.

Bagatell, C.J. and Bremner, W.J. (1996) Androgens in men - uses and abuses. New Engl $\mathrm{J}$ Med. 334(11):707-715.

Spencer, L., Bancroft, J. and Gamble, M. (2012) Tissue processing. Bancroft's Theory and Practice of Histological Techniques. 7nd ed. Amsterdam: Elsevier Health Sciences. Pp. 105-123.

Beattie, M.C., Adekola, L., Papadopoulos, V., Chen, H. and Zirkin, B.R. (2015) Leydig cell aging and hypogonadism. Exp Gerontol. 68:87-91.

Beck, K.F., Eberhardt, W., Walpen, S., Apel, M. and Pfeilschifter. J. (1998) Potentiation of nitric oxide synthase expression by superoxide in interleukin $1 \beta$ stimulated rat mesangial cells. FEBS Lett. 435(1):3538.

Benveniste, E.N. (1998) Cytokine actions in the central nervous system. Cytokine Growth Factor Rev. 9(3-4):259-275.

Bhasin, S., Cunningham, G.R., Hayes, F.J., Matsumoto, A.M., Snyder, .P.J., Swerdloff, R.S., et al. (2010) Testosterone therapy in men with androgen deficiency syndromes: an Endocrine Society clinical practice guideline. J Clin Endocrin Metab. 95(6):2536-2559.

Cappellano, G., Carecchio, M., Fleetwood, T., Magistrell,i L, Cantello R, Dianzani U, Comi C. (2013) Immunity and inflammation in neurodegenerative diseases. Am J Neurodegenerative Dis. 2(2):89.

Chen, H., Cangello, D., Benson, S., Folmer, J., Zhu, H., Trush, M.A., et al. (2001) Age-related increase in mitochondrial superoxide generation in the testosterone-producing cells of Brown Norway rat testes: relationship to reduced steroidogenic function?. Exp Gerontol. 36(8):1361-1373.

Cherrier, M.M., Asthana, S., Plymate, S., Baker, L., Matsumoto, A.M., Peskind, E., et al. (2001) Testosterone supplementation improves spatial and verbal memory in healthy older men. Neurol. 57(1):80-88.

Damaj, M.I. (2001) Influence of gender and sex hormones on nicotine acute pharmacological effects in mice. J Pharmacol Exp Therapeut. 296(1):132140.

Dewing, P., Shi, T., Horvath, S. and Vilain, E. (2003) Sexually dimorphic gene expression in mouse brain precedes gonadal differentiation. Mol Brain Res. 118(1-2):82-90.
Ferrini, R.L. and Barrett-Connor, E. (1998) Sex hormones and age: a cross-sectional study of testosterone and estradiol and their bioavailable fractions in community-dwelling men. Am J Epidemiol. 147(8):750-754.

Gonzalez-Forero, D. and Moreno-Lopez, B. (2014) Retrograde response in axotomized motoneurons: nitric oxide as a key player in triggering reversion toward a dedifferentiated phenotype. Neurosci. 283:138-165.

Gooren, L. and Asscheman, H. (2014) Sex reassignment: Endocrinological interventions in adults with gender dysphoria. In: Gender dysphoria and disorders of sex development. Springer, Boston, MA. Pp. 277-297.

Idris, A.I. (2012) Ovariectomy/orchidectomy in rodents. In: Bone Research Protocols. 2nd edn. Totowa, NJ: Humana Pre. Pp. 545-551

Jacome, L.F., Barateli, K., Buitrago, D., Lema, F., Frankfurt, M. and Luine, V.N. (2016) Gonadal hormones rapidly enhance spatial memory and increase hippocampal spine density in male rats. Endocrinol. 157(4):1357-1362.

Jang, E., Kim, J.H., Lee, S., Kim, J.H., Seo, J.W., Jin, M., et al. (2013) Phenotypic polarization of activated astrocytes: the critical role of lipocalin-2 in the classical inflammatory activation of astrocytes. J Immunol. 191(10):5204-5219.

Janowsky, J.S., Chavez, B. and Orwoll, E. (2000) Sex steroids modify working memory. J Cog Neurosci. 12(3):407-414.

Janowsky, J.S., Oviatt, S.K. and Orwoll, E.S. (1994) Testosterone influences spatial cognition in older men. Behav Neurosci. 108(2):325.

Jayaraman, A., Lent-Schochet, D. and Pike, C.J. (2014) Diet-induced obesity and low testosterone increase neuroinflammation and impair neural function. J Neuroinflammation. 11(1):162.

Katoh-Semba, R., Semba, R., Kato, H., Ueno, M., Arakawa, Y. and Kato, K. (1994) Regulation by androgen of levels of the $\beta$ subunit of nerve growth factor and its mRNA in selected regions of the mouse brain. J Neurochem. 62(6):2141-147.

Kluger, J., Roy, A., Chao, H.H. (2020) Androgen deprivation therapy and cognitive function in prostate cancer. Curr Oncol Reports. 22(3):24.

Madrigal, J.L., Hurtado, O., Moro, M.A., Lizasoain, I, Lorenzo, P., Castrillo, A., et al. (2002) The increase in TNF- $\alpha$ levels is implicated in NF-KB activation and inducible nitric oxide synthase expression in brain cortex after immobilization stress. Neuropsychopharmacol. 26(2):155-163.

May, J.M. (2000) How does ascorbic acid prevent endothelial dysfunction? Free Radical Biol Med. 28(9):1421-1429.

Medeiros,, R., Prediger, R.D., Passos, G.F., Pandolfo, P., Duarte, F.S., Franco, J.L., et al. (2007) Connecting TNF- $\alpha$ signaling pathways to iNOS expression in a mouse model of Alzheimer's disease: relevance for the behavioral and synaptic deficits 
induced by amyloid $\beta$ protein. J Neurosci. 27(20):5394-5404.

Mellon, S.H. and Deschepper, C.F. (1993) Neurosteroid biosynthesis: genes for adrenal steroidogenic enzymes are expressed in the brain. Brain Res. 629(2):283-292.

Morris, P.D. and Channer, K.S. (2012) Testosterone and cardiovascular disease in men. Asian $\mathrm{J}$ Androl. 14(3):428.

Nakamura, N, Fujita, H. and Kawata, M. (2002) Effects of gonadectomy on immunoreactivity for choline acetyltransferase in the cortex, hippocampus, and basal forebrain of adult male rats. Neurosci. 109(3):473-485.

Namiki, M., Akaza, H., Shimazui, T., Ito, N., Iwamoto, T., Baba, K., et al. (2008) Clinical practice manual for late-onset hypogonadism syndrome. Int $\mathrm{J}$ Urol. 15(5):377-388.

Okun, M.S., DeLong, M.R., Hanfelt, J., Gearing, M. and Levey, A. (2004) Plasma testosterone levels in Alzheimer and Parkinson diseases. Neurol. 62(3):411-413.

Pilati, N., Barker, M., Panteleimonitis, S., Donga, R. and Hamann, M. (2008). A rapid method combining Golgi and Nissl staining to study neuronal morphology and cytoarchitecture. J Histochem Cytochem, 56(6): 539-550.

Ridet, J.L., Privat, A., Malhotra, S.K, and Gage, F.H. (1997) Reactive astrocytes: cellular and molecular cues to biological function. Trends Neurosci. 20(12):570-577.

Rosa, S.D., Cirillo, P., Paglia, A., Sasso, L., Palma, V.D. and Chiariello, M. (2010) Reactive oxygen species and antioxidants in the pathophysiology of cardiovascular disease: Does the actual knowledge justify a clinical approach? Curr Vascular Pharmacol. 8(2):259-275.

Soreq, H., and Seidman, S. (2001) Acetylcholinesterase - new roles for an old actor. Nature Rev Neurosci. 2(4):294-302.

Streit, W.J., Mrak, R.E. and Griffin, W.S. (2004) Microglia and neuroinflammation: a pathological perspective. Journal of neuroinflammation. 1(1):1-4

Sunday, F.O., Dauda, S.P., Oladele, O.J., Sunday, O.Y., Abosede, A.S. and Faith, S.O. (2019) Moringa oleifera leaf extract potential in ameliorating MK-801induced schizophrenia. J Exp Clin Anat. 18(1):69.

Veldhuis, J.D., Keenan, D.M., Liu, P.Y., Iranmanesh, A., Takahashi, P.Y. and Nehra, A.X. (2009) The aging male hypothalamic-pituitary-gonadal axis: pulsatility and feedback. Mol Cellular Endocrinol. 299(1):14-22.

Vicencio, J., Estrada, M., Galvis, D., Bravo, R.E Contreras, A., Rotter, D., Lavandero, S. (2011) Anabolic androgenic steroids and intracellular calcium signaling: a mini review on mechanisms and physiological implications. Mini Rev Med Chem. 11(5):390-398.

Wiltink, J., Beutel, M.E., Brähler, E. and Weidner, W. (2009) Hypogonadism - related symptoms: development and evaluation of an empirically derived self-rating instrument (HRS 'hypogonadism related symptom scale'). Andrologia. 41(5):297-304.

Yaffe, K., Lui, L.Y., Zmuda, J. and Cauley, J. (2002) Sex hormones and cognitive function in older men. $\mathrm{J}$ Am Geriatrics Society. 50(4):707-712.

Yawson, E.O. and Akinola, O.B. (2021) Hippocampal cellular changes in androgen deprived insulin resistant rats. Metab Brain Dis. 36(5):1037-1048.

Cite as Abayomi, T.A., Tokunbo, O.S., Oyetunti, M., Yawson, E.O., Abayomi, O.A. and Ogunrinde, O.D. (2021) Ascorbic acid supplementation attenuates hippocampal neuronal damage in orchiectomized rats. Nig. J. Neurosci. 12(2):40-49. http://doi.org/10.47081/njn2021.12.2/001 